\title{
GIARDIASIS Y CRIPTOSPORIDIASIS EN CANINOS DE LOS DISTRI- TOS DEL CONO OESTE DE LIMA METROPOLITANA
}

\author{
Giardiasis And Cryptosporidiosis in Dogs of the Western Area of \\ Metropolitan Lima
}

\author{
Hernán Sotelo P. ${ }^{1}$, Amanda Chávez V. ${ }^{1}$, Eva Casas A. ${ }^{1}$, Rosa Pinedo V. ${ }^{1}$, \\ Néstor Falcón P. ${ }^{2}$
}

\section{Resumen}

\begin{abstract}
El objetivo del estudio fue determinar la prevalencia de Giardia spp y Cryptosporidium spp en caninos criados en los distritos del Cono Oeste de Lima Metropolitana, así como su asociación con las variables sexo, edad, estado físico de las heces, tipo de alimentación y permanencia en el hogar. Se recolectaron 300 muestras fecales de perros aparentemente sanos, de ambos sexos, diversas razas y con edades entre 1 mes a 12 años. Se utilizaron la técnicas de sedimentación espontánea para el diagnóstico de Giardia spp y la tinción de Ziehl-Neelsen modificado para Cryptosporidium spp. Se encontró una prevalencia de $16.7 \pm 4.0$ y $29.7 \pm 5.0 \%$ para Giardia spp y Cryptosporidium spp, respectivamente. La prevalencia de Giardia spp fue mayor en animales menores de 6 meses $(\mathrm{p}<0.05)$, mientras que animales mayores de 6 años mostraron frecuencias altas de Cryptosporidium spp $(\mathrm{p}<0.05)$. Así mismo, formas parasitarias de Giardia spp fueron detectadas con mayor frecuencia en heces sueltas que en heces normales $(\mathrm{p}<0.05)$. No se hallaron diferencias significativas entre la presencia de estos protozoos por efecto de las variables sexo, tipo de alimentación y permanencia en el hogar de los canes. Los resultados demuestran la existencia de una prevalencia moderada de Giardia spp y Cryptosporidium spp en la población canina de una importante zona urbana de Lima y su presencia en caninos podría constituir un serio problema para la Salud Pública, en especial a niños y personas inmunosuprimidas.
\end{abstract}

Palabras clave: Giardia spp, Cryptosporidium spp, diarrea, protozoo, zoonosis, prevalencia, perros, Ziehl-Neelsen modificado, sedimentación espontánea

\footnotetext{
${ }^{1}$ Laboratorio de Parasitología y Microbiología Veterinaria, Facultad de Medicina Veterinaria, Universidad Nacional Mayor de San Marcos, Lima

${ }^{2}$ Facultad de Veterinaria y Zootecnia, Universidad Peruana Cayetano Heredia, Lima
}

Recibido: 8 de marzo de 2012

Aceptado para publicación: 18 de febrero de 2013 
The aim of this study was to determine the prevalence of Giardia spp and Cryptosporidium spp in dogs reared in the western districts of Metropolitan Lima and the association with sex, type of diet, stools physical aspect and staying at home. For this, 300 fecal samples were collected from apparently healthy dogs of both sexes and various breeds between 1 month and 12 years of age. The spontaneous sedimentation technique was used for the diagnostics of Giardia spp and the Ziehl-Neelsen modified was used for Cryptosporidium spp. The prevalence was $16.7 \pm 4$ and $29.7 \pm 5.0 \%$ for Giardia spp and Cryptosporidium spp respectively. Younger animals showed higher prevalence of Giardia spp $(\mathrm{p}<0.05)$ whereas dogs older than 6 years showed higher prevalence of Cryptosporidium spp ( $<<0.05$ ). Also, Giardia spp was most commonly found in watery stools than in normal feces $(\mathrm{p}<0.05)$. None significant differences due to the presence of these protozoa were found in relation to sex, type of diet and staying at home. The results showed the presence of moderate prevalence of Giardia spp and Cryptosporidium spp in canine population of a major urban area of Lima. These dogs could be a serious problem for public health, especially children and immunosuppressed people.

Key words: Giardia spp, Cryptosporidium, diarrhea, protozoa, zoonotic, prevalence, dog, Ziehl-Neelsen modified, spontaneous sedimentation

\section{INTRODUCCIÓN}

Giardia spp y Cryptosporidium spp son parásitos de distribución mundial y de importancia en Salud Pública debido a que ocasionan una gastroenteritis de severidad variable, causando deterioro físico, desnutrición y retraso en el crecimiento y desarrollo, tanto en el hombre como en animales (Cordero del Campillo et al., 1999; Acha y Szyfres, 2003). La infección por estos agentes se observa con mayor frecuencia en individuos jóvenes, particularmente en zonas rurales de África, Asia y Sudamérica donde las condiciones medioambientales son favorables para su transmisión; siendo menos frecuentes en países industrializados (Acha y Szyfres, 2003).

La giardiasis es una parasitosis causada por el protozoo flagelado Giardia spp que se encuentra en el intestino delgado de perros, gatos, vacunos y otros animales domésticos, originando cuadros de síndrome de malabsorción y diarrea (Cordero del Campillo et al., 1999).
La criptosporidiasis es una enfermedad parasitaria de distribución cosmopolita, descrita en más de 170 especies de vertebrados y causada por protozoos del género Cryptosporidium (Ortega et al., 1999). Se han descrito 16 especies, incluyendo $C$. nasorum en peces, $C$. serpentis en reptiles y $C$. meleagridis en el intestino de aves (Fayer y Xiao, 2008). Dentro de estas, C. parvum es poco específico para su hospedero y ha sido asociado con episodios de diarrea en humanos, rumiantes, cerdos y otros mamíferos, y ocasionalmente en gallinas (Fayer y Xiao, 2008).

En animales de compañía, $C$. parvum es causante de graves cuadros entéricos, generalmente asociado a estados de inmunosupresión como el moquillo canino y la leucemia e inmunodeficiencia felina (Ortega et al., 1999). Además, se le encuentra asociado a virus entéricos (Rotavirus), bacterias (Campylobacter, Salmonella) y otros protozoos (Giardia). Ocasionalmente, se le ha encontrado en el estómago, vesícula biliar, hígado, páncreas, útero, tráquea, pulmones, 
corazón, vías urinarias y conjuntiva de terneros, cerdos y ratones infectados natural y experimentalmente (Ortega et al., 1999).

Estudios realizados en Lima Metropolitana indican prevalencias en perros de $25.4 \%$ a C. parvum y de $15.7 \%$ a Giardia spp (Romero et al., 2000) y $15.7 \%$ de perros positivos a Giardia spp (Zárate et al., 2003). Asimismo, en la provincia del Callao se obtuvo $9.4 \%$ de perros positivos a Giardia spp (Araujo et al., 2004). Tanto Giardia spp como Cryptosporidium spp son considerados de gran potencial zoonótico, pues el hombre puede infectarse al ingerir quistes y ooquistes provenientes de canes y otros animales (Robertson y Gjerde, 2001; Botero y Restrepo, 2006).

En el Perú, la giardiasis y la cryptosporidiasis se halla presente en los caninos, pero no existen datos actuales sobre el grado de infección en la población canina. El objetivo del estudio fue determinar la prevalencia de ambos parásitos en perros de varios distritos del Cono Oeste de Lima Metropolitana.

\section{Materiales y Métodos}

El estudio se llevó a cabo en canes domésticos, aparentemente sanos, de edad y raza diversas y de ambos sexos, cuyos propietarios residían en los distritos ubicados en el Cono Oeste de Lima Metropolitana (Breña, Jesús María, La Victoria, Lima, Lince, Magdalena del Mar, Miraflores, Pueblo Libre, San Borja, San Isidro, San Luis, San Miguel y Surquillo). El estudio se llevó a cabo entre agosto de 2009 y julio de 2010.

El tamaño muestral fue determinado mediante la fórmula para estimar una proporción (Daniel, 1996) y utilizando una prevalencia referencial de $25.4 \%$ (Romero et al., 2000). El tamaño mínimo resultante fue de 291 muestras; sin embargo, se colectaron 300 muestras. Este número representó el
$0.164 \%$ de la población total estimada de perros (182 994) de los distritos del cono Oeste de Lima Metropolitana, según el censo de la población humana (INEI, 2005).

Para la recolección de muestras se pidió la colaboración de propietarios de canes, a los cuales se les facilitó frascos plásticos rotulados y se les informó un día antes la forma de tomar las heces (aprox. $8 \mathrm{~g}$ ), libre del contacto de tierra y orina. La selección de los hogares se hizo en forma aleatoria. Las muestras fueron llevadas al Laboratorio de Parasitología de la Facultad de Medicina Veterinaria, Universidad Nacional Mayor de San Marcos, Lima, donde fueron guardadas en refrigeración. El análisis de las muestras se hizo mediante las pruebas de sedimentación espontánea y tinción de Ziehl-Neelsen modificada para diagnosticar la presencia de Giardia spp y Cryptosporidium spp, respectivamente (Rojas, 2004).

Las posibles asociaciones entre parásitos con las variables sexo, edad ( 1 a $6,>6$ a $12,>12$ a 72 y $>72$ meses), estado físico de las heces (formada, pastosa, diarreica), tipo de alimentación (casera, balanceado, mixta) y permanencia en el hogar (sí, no) se analizaron mediante la prueba de Chi cuadrado con un nivel de significancia de 0.05. Los datos fueron analizados utilizando el paquete estadístico SPSS v. 10.0 para Windows.

\section{Resultados}

La prevalencia de Giardia spp y Cryptosporidium spp en caninos de los distritos del Cono Oeste de Lima Metropolitana fue de $16.7 \pm 4.0$ y $29.7 \pm 5.0 \%$, respectivamente, con un nivel de confianza del $95 \%$ (Cuadro 1).

Se encontraron diferencias significativas entre grupos etarios, observándose que los animales más jóvenes son más propensos a estar infectados con Giardia spp ( $\mathrm{p}<0.05)$. Asimismo, se encontró que animales mayores de 72 meses tenían una mayor prevalen- 
Cuadro 1. Prevalencia de Giardia spp y Cryptosporidium spp en perros domésticos de los distritos del Cono Oeste de Lima Metropolitana, según sexo, edad, estado físico de las heces, tipo de alimentación y permanencia en el hogar (2009-2010)

\begin{tabular}{|c|c|c|c|c|c|}
\hline \multirow[b]{2}{*}{ Variables } & \multirow{2}{*}{$\begin{array}{l}\mathrm{N} .^{\circ} \text { de } \\
\text { muestras }\end{array}$} & \multicolumn{2}{|c|}{ Giardia spp } & \multicolumn{2}{|c|}{ Cryptosporidium spp } \\
\hline & & $\begin{array}{l}\text { Positivos } \\
\text { (n) }\end{array}$ & $\% \pm \mathrm{IC}^{1}$ & $\begin{array}{l}\text { Positivos } \\
\text { (n) }\end{array}$ & $\% \pm \mathrm{IC}^{1}$ \\
\hline \multicolumn{6}{|l|}{ Sexo } \\
\hline Macho & 178 & 36 & $20.2 \pm 6.0^{\mathrm{a}}$ & 54 & $30.3 \pm 7.0^{\mathrm{a}}$ \\
\hline Hembra & 122 & 14 & $11.5 \pm 6.0^{\mathrm{a}}$ & 35 & $28.7 \pm 8.0^{\mathrm{a}}$ \\
\hline \multicolumn{6}{|l|}{ Edad (meses) } \\
\hline 1 a 6 & 51 & 14 & $27.5 \pm 12.3^{\mathrm{a}}$ & 17 & $33.3 \pm 12.9^{\mathrm{ab}}$ \\
\hline$>6$ a 12 & 35 & 6 & $17.1 \pm 12.0^{\mathrm{ab}}$ & 13 & $37.1 \pm 16.0^{\mathrm{ab}}$ \\
\hline$>12$ a 72 & 159 & 27 & $17 \pm 6.0^{\mathrm{ab}}$ & 36 & $22.6 \pm 6.0^{\mathrm{a}}$ \\
\hline$>72$ & 55 & 3 & $5.5 \pm 6.0^{\mathrm{b}}$ & 23 & $41.8 \pm 13.0^{b}$ \\
\hline \multicolumn{6}{|c|}{ Estado físico de heces } \\
\hline Formada & 180 & 21 & $11.7 \pm 4.7^{\mathrm{a}}$ & 56 & $31.1 \pm 6.8^{\mathrm{a}}$ \\
\hline Pastosa & 94 & 21 & $22.4 \pm 8.4^{\mathrm{ab}}$ & 30 & $31.9 \pm 9.0^{\mathrm{a}}$ \\
\hline Diarreica & 26 & 8 & $30.8 \pm 17.8^{\mathrm{b}}$ & 3 & $11.5 \pm 12.3^{\mathrm{a}}$ \\
\hline \multicolumn{6}{|l|}{ Tipo de alimento } \\
\hline Casera & 62 & 10 & $16.1 \pm 9.0^{\mathrm{a}}$ & 20 & $32.3 \pm 11.6^{\mathrm{a}}$ \\
\hline Balanceado & 129 & 16 & $12.4 \pm 5.7^{\mathrm{a}}$ & 34 & $26.4 \pm 7.6^{\mathrm{a}}$ \\
\hline Mixta & 109 & 24 & $22.0 \pm 8.0^{\mathrm{a}}$ & 35 & $32.1 \pm 9.0^{\mathrm{a}}$ \\
\hline \multicolumn{6}{|c|}{ Permanencia en el hogar } \\
\hline Sí & 210 & 36 & $17.1 \pm 5.1^{\mathrm{a}}$ & 59 & $28.1 \pm 6.0^{\mathrm{a}}$ \\
\hline No & 90 & 14 & $15.6 \pm 7.5^{\mathrm{a}}$ & 30 & $33.3 \pm 9.7^{a}$ \\
\hline Total & 300 & 50 & $16.7 \pm 4.0$ & 89 & $29.7 \pm 5.0$ \\
\hline
\end{tabular}

cia de Cryptosporidium spp que animales de 12 a 72 meses $(\mathrm{p}<0.05)$. No hubo diferencias por efecto de las variables sexo, estado físico de las heces, tipo de alimento y permanencia en el hogar (Cuadro 1).

Se halló infección simultánea de ambos protozoos en 10 canes $(3.3 \pm 1.9 \%)$, donde siete animales presentaron heces pastosas.

\section{Discusión}

El $16.7 \%$ de prevalencia de Giardia spp hallada en el estudio fue similar a los resultados de Zárate et al. (2003) en caninos del Cono Sur de Lima Metropolitana (15.7\%). En ambos estudios se utilizó la técnica de sedimentación espontánea, mientras que 
Bazán et al. (2000), utilizando el método directo en 250 muestras fecales de caninos de vida intradomiciliaria del distrito limeño de San Juan de Lurigancho, encontraron una prevalencia baja $(0.8 \%)$. Es importante indicar que el método de sedimentación espontánea presenta mayor sensibilidad (70\%) que el examen directo (Larragán, 1993; Zárate et al., 2003). No obstante, en la Provincia Constitucional del Callao, con la de técnica de sedimentación espontánea se encontró una prevalencia de 9.4\% (Araujo et al., 2004).

Asimismo, reportes de zonas urbanas de otros países, empleando una sola muestra para el examen parasitológico, muestran resultados similares: $12 \%$ en España (Díaz et al., 1996), 16\% en Japón (Asano et al., 1991) y $21 \%$ en animales de refugio y criaderos en Perth, Australia (Swan $y$ Thompson, 1986); e incluso prevalencias superiores como el $36 \%$ en California, EEUU (Hahn et al., 1988). Estos datos demuestran la gran variabilidad de la presentación de Giardia, en parte debido a las características de la población canina, entorno ambiental y método de diagnóstico utilizado (Cordero del Campillo et al., 1999).

La prevalencia encontrada de $29.7 \%$ para el caso de Cryptosporidium spp es comparable con otros resultados en zonas urbanas (Romero et al., 2000) y rurales (Celis, 2010) del país empleando una técnica parasitológica similar. Sin embargo, las cifras de prevalencia de este parásito son inferiores en países vecinos como Chile $(1.9 \%$, Gorman et al., 2006) y Brasil (8.8\%, Lallo y Bondan, 2006), utilizando la misma técnica de diagnóstico. Es posible que estas diferencias sean debidas a la presencia de animales inmunocomprometidos y a la contaminación ambiental (Botero y Restrepo, 2006).

Otros factores que incrementan el riesgo de infección por el protozoario, tanto para el animal como las personas a su alrededor, serían el incremento de la población canina en zonas marginales y bajo nivel socio económico de la población, aunado a la falta de servicios básicos de agua y desagüe, desnutrición, mala higiene y hacinamiento (Botero y Restrepo, 2006). Además, la presencia de áreas verdes regadas con aguas servidas puede ser otro factor (Robertson y Gjerde, 2001), pues tanto Giardia spp como Cryptosporidium spp se difunden mejor en medio hídrico (Ortega et al., 1999).

La mayor susceptibilidad de cachorros de hasta seis meses de edad encontrada en el presente estudio (27.5\%) ha sido ampliamente demostrada en otros estudios (Hahn et al., 1988; Cordero del Campillo et al., 1999; Itoh et al., 2001; Zárate et al., 2003). Asimismo, un estudio en niños españoles de 6 a 10 años reporta que la frecuencia de presentación de Giardia spp disminuye con la mayor edad de los canes, lo cual se interpretaría como consecuencia de un cierto grado de inmunidad adquirida (Pérez et al., 1997).

Con respecto a la influencia de la edad en la presentación de Cryptosporidium spp, la mayor, aunque no significativa, tasa de prevalencia en animales menores de un año y la mayor prevalencia en animales mayores de 72 meses ( $\mathrm{p}<0.05)$, podría ser explicada por la mayor susceptibilidad de los animales jóvenes ante un pobre desarrollo inmunológico (Ortega et al., 1999; Acha y Zyfres, 2003), así como la baja capacidad de respuesta inmune en animales geriátricos (Cordero del Capillo et al., 1999); sin embargo, otros estudios no han encontrado una relación significativa entre edad y el hallazgo de Cryptosporidum spp en heces (Romero et al., 2000; Celis, 2010).

La relación entre el estado físico de las heces y la detección de Giardia spp fue significativa ( $\mathrm{p}<0.05)$. Canes positivos a Giardia spp presentaron una mayor frecuencia de heces diarreicas $(30.8 \%)$ y pastosas $(22.4 \%)$, coincidiendo con el estudio de Zárate et al. (2003), quienes indican una mayor probabilidad de hallar quistes y trofozoítos de Giardia spp en heces sueltas que en heces normales. Similar resultado fue obtenido por Itoh et al. (2001) en caninos domésticos del Japón. En 
ese sentido, se debe tener en cuenta que la diarrea es un trastorno gastrointestinal que acompaña a diversas enfermedades; sin embargo, la giardiosis clínica que cursa con diarrea o heces que no son aparentemente normales es generalmente de tipo recurrente $o$ crónica y los animales adelgazan a pesar que no pierden el apetito.

El Cryptosporidium spp parece no afectar el estado físico de las heces. El canino llega a infectarse sin desarrollar la enfermedad (Lloyd y Smith, 1997); no obstante, infecciones naturales con Cryptosporidum spp pueden llegan a causar cuadros diarreicos, especialmente en animales jóvenes, inmunosuprimidos o que cursan con otras infecciones bacterianas o virales que pueden provocar mortalidad (Romero et al., 2000). En los últimos años se reportaron hospederos de Cryptosporidium en más de 150 mamíferos, basado en el parecido microscópico de los ooquistes de $C$. parvum en las heces (Fayer, 2004). En muchos casos C. parvum fue el nombre de la especie por defecto sin la confirmación molecular y biológica (Fayer, 2010). En la actualidad se reconoce la presencia en caninos de $C$. canis y en el hombre de $C$. hominis; siendo $C$. canis considerada como zoonosis poco frecuente (Bowman, 2011).

El sexo, tipo de alimentación y permanencia en el hogar no fueron factores importantes en la presentación de estas enfermedades; resultados que coinciden con otros estudios en Giardia (Cordero del Campillo et al., 1999; Zárate et al., 2003) y Cryptosporidium (Romero et al., 2000; Lallo y Bondan, 2006). Sin embargo, OliveiraSequeira et al. (2002) encontraron una mayor prevalencia de Giardia spp en perros callejeros de la ciudad de Sao Paulo.

Finalmente, los canes que desarrollaron una infección mixta (3.3\%) tendrían una mayor probabilidad de manifestar sintomatología clínica que aquellos canes que solo cursan con un agente (Olivera-Sequeira et al., 2002).

\section{Conclusiones}

- La prevalencia de Giardia spp y Cryptosporidium spp en caninos domésticos del Cono Oeste de Lima Metropolitana fue de $16.7 \pm 4.0$ y $29.7 \pm$ $5.0 \%$ mediante las técnicas de sedimentación espontánea y Ziehl-Neelsen modificado, respectivamente.

- Las variables edad y el estado físico en el caso de Giardia spp y la edad en el caso de Cryptosporidium spp mostraron asociación estadística con la enfermedad $(\mathrm{p}<0.05)$.

\section{Literatura Citada}

1. Acha PN, Szyfres B. 2003. Zoonosis y enfermedades transmisibles comunes al hombre y a los animales. $3^{\text {a }}$ ed. Washington: OPS. 398 p.

2. Araujo W, Chávez A, Casas E, Falcón N. 2004. Prevalencia de Giardia sp. en Canis familiaris de la Provincia Constitucional del Callao. Rev Inv Vet Perú 15: 145-150.

3. Asano R, Hokari S, Murasugi E, Arracima Y, Kubo N, Kawano K. 1991. Studies on the giardiasis as the zoonosis II. Giardiasis in $\operatorname{dogs}$ and cats. Kansenshogaku Zasshi 65: 157-161.

4. Bazán H, Castillo Y, Salazar R, Sáez G. 2000. Enteroparásitos en Canis familiaris de S.J.L. En: Libro de Resúmenes IV Congreso Peruano de Parasitología. Lima, Perú.

5. Botero D, Restrepo M. 2006. Parasitosis humanas. $4^{\circ}$ ed. Bogotá, Colombia: Corporación para Investigaciones Biológicas. 506 p.

6. Bowman DD. 2011. Georgis. Parasitología para Veterinarios. $9^{\circ}$ ed. New York:. Elsevier. 453 p.

7. Celis NN. 2010. Criptosporidiasis en caninos criados en comunidades campesinas de tres distritos del departamento de Puno. Tesis de Médico Veterinario. 
Lima: Univ Nacional Mayor de San Marcos. 48 p.

8. Cordero del Campillo M, RojoVásquez FA, Martínez AR, Sánchez MC, Hernández S, Navarrete I, Diez $\boldsymbol{P}$, et al. 1999. Parasitología veterinaria. Madrid: McGraw-Hill Interamericana. $968 \mathrm{p}$.

9. Daniel D. 1996. Bioestadística. Base para el análisis de las ciencias de la salud. $5^{\circ}$ ed. México: Limusa. 878 p.

10. Díaz V, Campos M, Lozano J, Manas I, Gonzales J. 1996. Aspects of animal giardiosis in Granada province. Vet Parasitol 64: 171-176.

11. Fayer R, Xiao L. 2008. Cryptosporidium and cryptosporidiosis. $2^{\text {nd }}$ ed. Florida, USA: CRC Press. 565 p.

12. Fayer R. 2004. Cryptosporidium: a water-borne zoonotic parasite. Vet Parasitol 126: 37-56.

13. Fayer R. 2010. Taxonomy and species delimitation in Cryptosporidium. Exp Parasitol 124: 90-97.

14. Gorman T, Soto A, Alcalino H. 2006. Parasitismo gastrointestinal en perros de comunas de Santiago de diferente nivel socioeconómico Parasitol Latinoam 61: 126-132.

15. Hahn N, Glaser C, Hird D, Hirsh D. 1988. Prevalence of Giardia in the feces of pups. J Am Vet Med Assoc 192: 1428-1429.

16. INEI [Instituto Nacional de Estadística e Informática]. 2005. Censo, población y vivienda 2005. Población total, por sexo y lugar de empadronamiento, según distrito de Lima Metropolitana. Lima, Perú. [Internet], [15 julio 2009]. Disponible en: http://www.inei.gob.pe

17. Itoh N, Muraoka N, Aoki M, Itagaki T. 2001. Prevalencia of Giardia lamblia in household dogs. Kansenshogaku Zasshi 75: 671-677.

18. Lallo MA, Bondan EF. 2006. Prevalência de Cryptosporidium spp. em cães de instituições da cidade de São Paulo. Rev Saúde Públ, São Paulo 1(2): 20-22.
19. Larragán M. 1993. Comparación de los principales métodos diagnósticos para enteroparásitos. Tesis de Médico. Lima: Facultad de Medicina Humana, Univ Peruana Cayetano Heredia. $50 \mathrm{p}$.

20. Lloyd S, Smith J. 1997. Pattern of Cryptosporidium parvum oocyst excretion by experimentally infected dogs. Int J Parasitol 27: 799-801.

21. Oliveira-Sequeira TCG, Amarante AFT, Ferrari TB, Nunes LC. 2002. Prevalence of intestinal parasites in dogs from São Paulo State, Brazil. Vet Parasitol 103: 19-27.

22. Ortega ML, Gómez M, Rojo FA. 1999. Criptosporidiosis. En: Cordero del Campillo M, Rojo FA, Martínez AR, Sánchez MC, Hernández S, Navarrete I, et al. (eds). Parasitología veterinaria. Madrid: McGraw-Hill Interamericana. p 213-221.

23. Pérez AC, Ariza AC, Úbeda OJ, Guevara BD, De Rojas AM, Lozano $S C$. 1997. Epidemiología del parasitismo intestinal infantil en el valle del Guadalquivir, España. Rev Esp Salud Públ 71:547-552.

24. Robertson LJ, Gjerde B. 2001. Occurrence of Cryptosporidium oocysts and Giardia cysts in raw waters in Norway. Scand J Public Hlth 29: 200-207.

25. Rojas M. 2004. Nosoparasitosis de los rumiantes domésticos peruanos. $2^{\mathrm{a}}$ ed. Lima: Martegraf $146 \mathrm{p}$.

26. Romero M, Chávez A, Casas E. 2000. Determinación de la presencia de Cryptosporidium parvum y Cyclospora spp. en caninos domésticos (Canis familiaris) en los distritos de Lima Metropolitana. Rev Inv Vet Perú 11: 26-31.

27. Swan J, Thompson R. 1986. The prevalence of Giardia in dogs and cats in Perth, Western Australia. Aust Vet J 63: 110-112.

28. Zárate D, Chávez A, Casas E, Falcón N. 2003. Prevalencia de Giardia sp. en canes de los distritos del cono sur de Lima Metropolitana. Rev Inv Vet Perú 14: 134-139. 